28 | 2002

Histoire de l'enseignement du français langue

étrangère ou seconde dans le bassin méditerranéen.

Volume 2

\title{
Echos phonétiques en Sicile entre XIXe et XXe siècles
}

\author{
Enrica Galazzi
}

\section{OpenEdition \\ Journals}

\section{Édition électronique}

URL : https://journals.openedition.org/dhfles/2626

DOI : $10.4000 /$ dhfles.2626

ISSN : 2221-4038

\section{Éditeur}

Société Internationale pour l'Histoire du Français Langue Étrangère ou Seconde

Édition imprimée

Date de publication : 1 juin 2002

Pagination : 13-31

ISSN : 0992-7654

Référence électronique

Enrica Galazzi, «Echos phonétiques en Sicile entre XIXe et XXe siècles », Documents pour I'histoire du français langue étrangère ou seconde [En ligne], 28 | 2002, mis en ligne le 31 janvier 2014, consulté le 27 mai 2021. URL : http://journals.openedition.org/dhfles/2626 ; DOI : https://doi.org/10.4000/dhfles. 2626

Ce document a été généré automatiquement le 27 mai 2021

(c) SIHFLES 


\title{
Echos phonétiques en Sicile entre XIXe et XXe siècles
}

\author{
Enrica Galazzi
}

1 Dans mon parcours de recherche autour de « phonétique ét enseignement du français ", mon regard a été attiré par la Sicile, pays de culture et de contrastes, qui ne cesse d'étonner le chercheur par son ouverture au monde en dépit de ses frontières géographiques naturelles qui paraissent la délimiter en espace clos.

2 En 1861 (juste après l'unification de l'Italie) le taux d'analphabétisme en Sicile dépasse largement la moyenne nationale (78\%) se situant entre $88,7 \%$ et $91,7 \%$ (Vaccina 1964 : 75 ; Lillo 1995 : 143).

3 Le système éducatif légué par le Royaume des deux Siciles est désastreux. Dans ce panorama désolant, on remarque une tradition dans l'édition des ouvrages scolaires. Deux villes sont tout particulièrement concernées : Messine et Palerme. Pour la première, la publication d'une grammaire du français remonte à 1675 , tandis que pour la deuxième, les manuels recensés sont plus nombreux, mais le premier ne date que de 1817 (Minerva-Pellandra 1991). Tous ces manuels consacrent un chapitre aux sons bien avant que ceux-ci n'aient fait l'objet d'une analyse et d'un classement scientifiques. Les auteurs sont, généralement, des enseignants de français (ou de plusieurs langues comme c'était la coutume à l'époque); les ouvrages s'adressent au public scolaire de l'île, une élite si on considère le taux des illettrés, et de l'ensemble du pays.

Dans cette communication seront pris en considération uniquement les traités consacrés à la prononciation, car il y en a qui se publient en Sicile au carrefour des deux siècles.

La période qui nous intéresse se situe après 1875 , date de naissance de la phonétique, et est marquée à l'échelle internationale par des événements essentiels pour la didactique: en 1886, la fondation de l'Association Phonétique Internationale et de son bulletin « le Maître Phonétique»; en août de la même année, le Congrès Philologique de Stockholm dominé par les thèses des phonéticiens : Passy, Jespersen, Sweet, Viëtor et les linguistes scandinaves. L'enseignement de la langue parlée étant reconnu comme prioritaire, la 
phonétique est destinée à jouer un rôle majeur dans cette révolution méthodologique qui aboutit à l'adoption de la méthode directe (Puren 1988).

6 Ce qui est frappant c'est que, de 1897 à 1912, quatre manuels de phonétique française sont publiés en Sicile dans quatre villes différentes : Messine, Catane, Caltanissetta, Palerme, tandis qu'on n'en dénombre pour ainsi dire pas autant sur l'ensemble du pays dans le même laps de temps. Quelle est l'origine et la formation des auteurs de ces ouvrages ? Comment expliquer l'avancée des idées phonétiques en Sicile alors que l'Italie n'avait fait aucun progrès dans ce domaine scientifique ? Par quels ouvrages et/ ou par quels personnages les nouvelles idées sont-elles arrivées dans l'île?

\section{Hypothèses}

\section{Le moment historique}

7 L'avènement de la méthode directe dans l'Europe tout entière provoque un large débat et la mise en cause de la méthode traditionnelle (dite de grammaire-traduction).

En Italie, la circulaire du Ministre Bianchi en 1905, et quelques autres précédemment, mettent l'accent sur l'importance de l'oral mais l'enseignement reste longtemps encore ancré dans la tradition grammaticale.

9 Dans le cursus scolaire, les filières classiques, les plus prestigieuses, se retranchaient derrière le rôle formateur des langues mortes opposé à la valeur purement instrumentale reconnue aux langues vivantes. C'était un véritable affrontement, dans tous les pays, entre les classiques et les modernes.

Les Scuole tecniche et Istituti tecnici étaient à l'époque les seuls établissements scolaires où on enseignait les langues vivantes. Ces écoles proposaient un enseignement fonctionnel avant la lettre, de plusieurs langues.

11 Dans les écoles commerciales les langues vivantes devaient « servir à la vie des affaires internationales »(Lovera 1916). Par conséquent, outre la langue et la littérature, l'enseignement devait envisager les manifestations les plus importantes de la vie sociale et économique du pays étranger.

Dans les Ginnasi l'enseignement du français deviendra optionnel en 1889 et obligatoire à partir de 1892 (Pellandra $1991: 221$ ).

\section{Intérêt de la Sicile pour la langue et la littérature françaises}

13 L'histoire nous apprend l'attachement des Siciliens à la langue et à la culture françaises. Preuve en sont, depuis le régime napoléonien, la tradition dans la rédaction bilingue des journaux les plus diffusés (par exemple le Corriere di Napoli e di Sicilia), la floraison des traductions de romans français et l'intense production locale de manuels pour l'apprentissage de la langue. Il s'agit d'une domination politique et culturelle dont les marques ne furent que partiellement effacées par les Bourbons. Même après l'unification de la péninsule, la langue française continua à occuper une place privilégiée (Vitale 2001).

14 Cet attachement des siciliens au français se retrouve également dans la critique littéraire. Nous ne prendrons qu'un seul exemple. La première revue symboliste italienne paraît à Messine (directeur Enrico Cardile), témoignage parmi d'autres d'un 
lien privilégié avec la poésie transalpine. Ars Nova, revue " di lettere scienze e arti ", parue entre 1901 et 1905 (dir. Giuseppe Arrosto) publie des pages sur le symbolisme « messinese ». Nourris de Baudelaire, Mallarmé, Verlaine, les poètes et les francisants présents à Messine dans la première moitié du $\mathrm{XX}^{\mathrm{e}}$ siècle sont nombreux : A. Toscano, Giuseppe Rino, Giovanni Calabro, U. Saffiotti, Antonio Restori, Glauco Natoli, Enrico Maria Camagna, Luca Pignato (Cf. Massimo e Sergio Palumbo, «Forse fu il vento di Sicilia ? », in AA.VV. 2000 : 13). En particulier, Natoli, Pignato et Calabro enseignaient la langue et la littérature françaises à l'Université et traduisaient les poètes symbolistes (cf. Adamo, sous presse).

15 A l'époque, la connaissance, la lecture, la traduction des œuvres littéraires étaient, beaucoup plus que de nos jours, le vecteur d'une culture et d'une langue.

\section{Présence de personnalités marquantes}

16 La diffusion des idées nouvelles venant de l'étranger, le vaste débat autour de l'enseignement des langues, de la formation et du recrutement des enseignants sont sans doute liés à un humus culturel particulièrement réceptif. Une société cosmopolite qui avait su intégrer un grand nombre d'étrangers ne pouvait qu'être ouverte sur le monde.

17 Quelques-uns des noms que nous avons croisés méritent qu'on s'y attarde.

18 *Giovanni Bonfiglio, professeur à l'école des Mines, à l'école Technique et au Lycée de Caltanissetta, Giovanni (Jean) Bonfiglio est au centre d'initiatives multiples.

19 Auteur de nombreux manuels destinés à l'enseignement de la langue française à différents niveaux, d'un Dictionnaire des Homonymes, d'un Dictionnaire contrastif italien/ français portant sur les différences syntaxiques entre les deux langues, d'un Précis de l'histoire de la littérature française, Bonfiglio fonde et dirige L'Ape (Lentini puis Caltanissetta, 1892-1913, discontinue, paraît le 15 de chaque mois de l'année scolaire) et Le Parisien " giornale pratico di lingua francese. »

*Romeo Lovera, directeur du Bollettino di Filologia Moderna, membre de l'Association Phonétique Internationale, Conseiller de cette même Association de 1904 à 1910 (à côté des grands initiateurs de la Réforme, notamment Paul Passy, Wilhelm Viëtor, Otto Jespersen), premier président de l'Association italienne des professeurs de langues étrangères fondée en 1903 ; a fait de nombreux séjours à l'étranger. En 1897 il participe au cours d'été de Marbourg où il assiste, entre autres, aux expériences phonétiques de Viëtor et de l'Abbé Rousselot. En 1904, à Marbourg, il inaugure les Conférences italiennes pendant les célèbres cours d'été organisés dans cette ville.

21 Lovera bénéficiait d'un grand prestige à l'intérieur de l'Association Phonétique Internationale puisque aux élections de 1907-8 il avait obtenu 71 voix se plaçant ainsi juste derrière les grands ténors (Passy : 99 voix ; Viëtor : 96 ; Jespersen : 86 ; Jones : 76) tandis que d'autres noms qui ont survécu au temps n'avaient pas fait mieux (ZündBurguet : 17 ; Panconcelli-Calzia : 13).

22 Polyvalent et polyglotte (il connaît le français, l'allemand, le roumain, le grec moderne), Lovera est un personnage-clé dont le profil reste à tracer. Il débarque à Palerme en octobre 1904 avec une expérience énorme d'enseignement, de formation, de gestion. Il y est appelé pour diriger la Scuola di Commercio. A partir de cette date, le Bollettino di Filologia Moderna dont il était le fondateur et l'âme est rédigé à Palerme. 
*Gaetano Salvemini, jeune professeur d'histoire à l'Université de Messine, qui deviendra un célèbre historien. Il participe au débat orageux sur les méthodes d'enseignement et la formation des enseignants qui bat son plein entre 1903 et 1909. Il s'exprime durement sur les concours de recrutement, les «Abilitazioni » et les enseignants de L2 qu'il définit «fortunali o trafficanti », dépourvus de toute formation scientifique. Ce qui provoqua des réactions indignées dans le Bollettino di Filologia Moderna (1903: 15-16).

24 *Antonio Restori, professeur d'Histoire comparée des littératures néolatines à Messine puis à Gênes et Président de la Faculté des Lettres de Messine en 1904-1905. Musicologue, philologue, auteur d'ouvrages sur les littératures espagnole et provençale, membre de différentes académies italiennes et étrangères (la Real Academia española et les Fébriles en France), Restori a publié de nombreux ouvrages parmi lesquels, en 1892, Note fonetiche sui parlari dell'alta valle di Magra, ce qui prouve son intérêt pour la phonétique et explique sans doute son inscription à l'Association Phonétique Internationale à partir de 1904.

\section{Rôle des revues}

Les nombreuses revues éditées en Sicile dans la période qui nous intéresse sont un indice supplémentaire de la vivacité culturelle et des ferments d'innovation qui germent dans l'île.

${ }^{*}{ }^{*}$ 'APE, periodico scolastico di lingua francese (revue scolaire de langue française) fondée en 1892 par Giovanni Bonfiglio, professeur à l'École des Mines, à l'École Technique et au Lycée de Caltanissetta. (1892-94 ; 1902-1906 ; 1908-1913) (Minerva 1996 : 192 ; Pellandra 1992 : 28). D'abord vouée à des tâches linguistiques (grammaticales) essentiellement pratiques, au tournant du siècle la revue se met à l'heure du jour et attiche en sous-titre " Revue de philologie et de littérature. " Dès lors, elle accueille aussi bien " la vieille langue » que la langue vivante, prévoit une galerie des poètes contemporains sans pour autant ignorer la Chanson de Roland (n. 1,15 oct. 1909), dans le seul but d'être utile. On y aborde également les aspects méthodologiques essentiels de la Réforme de l'enseignement des langues ( $L a$ réforme de l'enseignement des langues étrangères en Allemagne, APE 1902, n. 1, n. 2 et n. 3 ; La méthode directe, Ibid. 1904, n.6). En 1903, dans le numéro 3 du périodique, paraît un article de Paul Passy sur la méthode d'imitation raisonnée.

La partie méthodologique, réservée à l'enseignant, est suivie d'une partie didactique comprenant essentiellement des exercices de traduction, des dictées et des lectures (contes et nouvelles et un feuilleton). Aucune transcription phonétique n'est utilisée.

** Le Parisien " giornale pratico di lingua francese » (8 pages, 10 cent, parait tous les 10 jours, Caltanissetta 191 I-?, directeur Jean Bonfiglio).

29 «Le Parisien » est consacré à la pratique de la langue usuelle : dialogues familiers, exercices dialogiques, connaissances utiles, dialogues pour les dames... et à l'entraînement / révision grammaticale, à des exercices progressifs de thème /version, à la correspondance commerciale et familière. A noter la présence des corrigés et des Récréations.

30 Quant au traitement des textes de lecture présentés sur deux colonnes, en français et en italien, la transcription n'adopte pas l'A.RI. mais elle reste figurée (dans la vieille 
tradition scolaire) à l'aide des lettres telles qu'elles se prononcent en langue maternelle.

31 En dépit de ce choix tout à fait inadéquat, on constate une grande sensibilité aux phénomènes oraux tels que l'enchaînement. La resyllabation est correctement indiquée (ce qui n'arrive pas souvent!), par exemple $:$ il a donné = i-la-donné $;$ mais pas toujours la sonorisation en cas de liaison $:$ Fais entrer $=$ fé-santré $;$ Vous avez $=$ vu-savé (d'après Les aventures de deux étudiants. XLI Les potins, «Le Parisien » 11 mai 1911). Le manque d'outils de représentation des phénomènes de l'oral n'empêche pas l'intuition de ses spécificités. Tout au moins, pas toujours.

** Bollettino di Filologia Moderna (Salò, 1894-95 ; Venezia 1901-1909) fondé et dirigé par Romeo Lovera ; édité à Palerme après 1904.

Après une première parution, vite arrêtée à cause de l'incompréhension des collègues qui étaient arrivés jusqu'à renvoyer le Bulletin tellement il était en avance par rapport à la situation italienne, Romeo Lovera reprend de plus belle et devient le champion de la Réforme en Italie. En 1901 dans le « Bollettino » ses articles illustrent la méthode intuitive. Dans la même revue, qui se veut le rapporteur des événements à l'échelle européenne, les lecteurs ont droit à des comptes-rendus des ouvrages de Passy, Sweet, Nyrop, Zünd-Burguet, et au signalement des plus célèbres cours de vacances (entre autres ceux de Marbourg et de Grenoble).

En janvier 1905, depuis Palerme, Lovera s'adresse aux lecteurs du Bollettino pour confirmer son programme d'action pédagogique. Il affirme que les enseignants italiens n'ont pas su tirer parti des avantages qu'offre la phonétique. Cette lacune a fait l'objet d'articles nombreux (Cf. en particulier «BFM »n. 2, 1902). Étant donné le retard accumulé et le manque de structures de formation, Lovera se propose de consacrer à la phonétique " pratique " des articles simples, clairs, contenant des applications didactiques pour la classe («BFM » Janvier 1905, Ai lettori).

Bien plus que les deux revues précédemment citées, le BFM se fait l'écho des débats internationaux auxquels Lovera participe activement au sein de l'Association Phonétique Internationale. En outre, il est caractérisé par une dimension plurilingue qui touche l'ensemble des enseignants de langues vivantes dont il s'est fait le porteparole et auxquels il offre une tribune de débat et d'échange. Le français, l'allemand, l'anglais et l'espagnol font l'objet d'articles théoriques et pratiques.

**Nuovi doveri, revue fondée en 1907 par Giuseppe Lombardo-Radice, éditée à Palerme. Tout comme le "BFM ", cette revue a joué un rôle de premier plan dans le débat sur le renouvellement des études linguistiques et littéraires en Italie en donnant la parole aux législateurs, aux spécialistes de ces disciplines, aux pédagogues les plus réputés, aux maitres à penser de la culture italienne. Y ont collaboré des spécialistes italiens et étrangers. Elle propose des enquêtes, par ex.:

Comment les L2 sont-elles enseignées en Italie (1908) ? et dans les autres pays européens ? (1909, coordonné par Bruno Flury du R. Istituto Tecnico de Catania).

Pour la méthode directe prennent lu parole Piero Toldo (1907) cl Federico Garlanda (1909), tandis que Benedetto Croce y défend l'école « classique » (1909).

\section{Les traités de phonétique française publiés en Sicile après 1875}

Nous prendrons en considération cinq ouvrages parus en Sicile dans la période considérée. 

d'Italie. Précédé d'un traité complet de prononciation (1897) est intéressant à bien des égards : pour le public sélectionné mais aussi pour le but déclaré : accroître la culture générale et technique des élèves de l'enseignement secondaire et contribuer à l'acquisition parfaite de la langue dans toutes ses parties constitutives. Pour ce faire, trois sections composent l'ouvrage : I. Partie Littéraire ; II. Partie Commerciale ; III. Partie Scientifique.

\section{l'enseignement spécial des sections de commerce.} scientifiques et inspirées par les progrès techniques de l'époque.

L'importance accordée à la littérature dans la formation culturelle des étudiants des filières commerciales sera théorisée de manière explicite par R. Lovera dans sa Leçon d'ouverture à l'Institut des hautes études commerciales de Turin le 15 mars 1916. Après de nombreuses années passées dans différentes écoles de commerce de la péninsule, il écrivait ainsi :

Dans une école de commerce il est de toute importance de faire aller de pair avec

l'étude philologique, littéraire et technique, celle du pays et du monde de la France,

ce que les Allemands appellent les Realien (Lovera $1916: 7$ ).

La centralité reconnue aux aspects fonctionnels de l'enseignement linguistique (les relations internationales, le commerce, la société) ne remettait pas en cause la priorité de la prononciation qui apparaissait comme une sorte de pré-requis. Par ailleurs, il n'était nullement question du regrettable divorce auquel nous assistons aujourd'hui entre langue et littérature.

Pour en revenir à la phonétique (pp. 1-48), son traitement chez Deodato est tout à fait conforme à la tradition des manuels scolaires : les lettres sont le point de départ pour arriver aux sons. Pour chaque cas, l'auteur propose une liste de phrases hétérogènes et décousues (Applicazione) souvent teintées de moralisme où on loue les vertus et on fustige les vices.

Il n'y a aucune tentative de transcription sauf l'habituel tiret pour indiquer la liaison. La prononciation est indiquée à l'aide des graphèmes de la langue maternelle.

Les pages 20 à 47 sont consacrées à "Regole particolari ed eccezioni. » Suivant l'ordre alphabétique, l'auteur passe en revue le comportement capricieux des différentes « lettres ».

La lecture de ce chapitre donne l'impression fâcheuse et décourageante que la prononciation $\mathrm{du}$ français n'est qu'une somme de cas spéciaux n'obéissant pratiquement à aucune règle. Quant à son efficacité pédagogique, ce traité n'apporte aucun élément nouveau qui puisse aider l'enseignant et l'apprenant. Je suppose qu'à cette date les idées des jeunes phonéticiens n'avaient pas encore traversé les Alpes.

C'est au tournant du siècle que les choses ont l'air de changer.

La parution du Manuale di fonetica francese de Carlo Vincent (Catane 1906) est signalée dans le Maitre Phonétique de janvier -février 1907.

49 Nous avons glané, au fil des pages du Maître phonétique quelques bribes de l'histoire personnelle et professionnelle de Vincent. 

nouveaux membres dans le MF février-mars 1905), alors qu'il enseigne au Regio Istituto Tecnico de Reggio Calabria. En 1906 il exercera ses fonctions au Regio Istituto Tecnico de Catane.

$$
\text { électeurs du Conseil de l'Association Phonétique (MF, nov.-déc. } 1907 \text { : 107), mais, }
$$$$
\text { En 1907, il figure avec Lovera parmi les nombreuses candidatures proposées aux }
$$
contrairement à Lovera, il ne sera pas élu (MF, janv.-févr. 1908 : 29-30).

Dans son Manuale di fonetica francese, Vincent, qui est au courant des actions menées par l'Association Phonétique Internationale, déclare s'inspirer des ouvrages récents de Kristopher Nyrop, Paul Passy, Michaëlis-Passy, Arsène Darmesteter, Adolphe ZündBurguet et se définit comme un " subversif " qui verrait volontiers l'alphabet phonétique remplacer l'orthographe étymologique. Cette position ne va pas sans rappeler le jugement sévère porté par Michel Bréal sur les " extrémistes», des fanatiques qui n'hésitaient pas à s'attaquer au patrimoine culturel français. 
plan pédagogique. Peu adéquat au travail en classe, l'ouvrage est, d'après Naef, tout aussi inadéquat aux besoins des enseignants qui ont le droit et les capacités de puiser directement aux sources citées par Vincent. En particulier, il relève des incohérences dans le choix des prononciations (tantôt désuètes, tantôt excessivement modernes) et s'en tient rigoureusement au principe du modèle unique à proposer dans la classe. Notons en passant que ce souci de rigueur méthodologique (tout à fait illusoire, d'ailleurs) va à rencontre des positions des « jeunes phonéticiens » qui, tout en essayant d'éviter soigneusement les « inconséquences de transcription » (c'est-à-dire le mélange de prononciations appartenant à de styles différents) n'en étaient pas moins convaincus de l'intérêt de faire entendre ce qui est réellement prononcé dans différentes situations de parole et non ce qu'on croit qu'on devrait prononcer. (cf. à ce propos P. Passy, « Soyons conséquents ! », Maître Phonétique décembre 1906 : 127-128).

Dans sa réponse, Carlo Vincent, sans jamais quitter le ton courtois et sans aucune acrimonie, révèle un accord de fond et certaines divergences (Cf. Carlo Vincent, Correspondance, "A proposito d'una recensione ", Maître Phonétique mars-avril 1908, 51-53).

62 En effet, Vincent a personnellement expérimenté l'inefficacité pédagogique de son ouvrage écrit avec tant d'enthousiasme. Ayant distribué 140 exemplaires gratuits à ses élèves de la première classe de l'Institut technique de l'année scolaire précédente dans l'illusion d'améliorer leur prononciation barbare, il a constaté que moins de dix pour cent en avaient profité, si bien que pour l'année en cours il n'avait pas répété l'expérience se limitant à suivre les programmes, sans plus.

63 Quant aux contrastes, Vincent relève un excès de sévérité chez Naef (qui arrive à considérer comme fautes de prononciation d'évidentes coquilles) et une contradiction évidente : comment peut-on en effet insister sur l'indifférence des Italiens face à la phonétique et, toutefois, considérer comme offensant pour les enseignants un ouvrage d'initiation à cette discipline?

Quant au modèle de prononciation, Vincent réaffirme la nécessité de prendre garde à la valeur morphologique des oppositions vocaliques (dans le cas précis l'opposition [E] fermé vs ouvert que Naef considère comme « illusoire ») et est de l'avis - très judicieux qu'un enseignant ne peut pas enseigner une prononciation autre que la sienne. Et cela aussi bien en langue maternelle que dans les langues étrangères. Le cas de l'italien est révélateur et les deux textes de Naef et Vincent, rédigés en API, en sont un témoignage éloquent.

Dans Le Maître Phonétique de nov.-déc. 1912, dans la rubrique Comptes rendus, Paul Passy s'exprime brièvement sur l'ouvrage de Alberto Ferrante Petite phonétique de la langue française à l'usage des Italiens (1912). Il critique l'emploi d'un alphabet personnel qui n'est guère bien choisi. En tant que travail de vulgarisation à l'usage des écoles italiennes il estime qu' " on y trouve pas mal de bonnes choses, mais aussi beaucoup de longueurs, des assertions discutables ou inexactes (page 59 : "les désinences des mots seul et seule, vis et vice, pareil et pareille, finir et empire, devoir et victoire, amer et colère, ne sont pas tout à fait les mêmes" : c'est du pur roman) »(pp. 139-140). L'impression générale de Passy est que l'auteur " a étudié beaucoup de livres, mais n'a pas assez observé par lui-même » (p. 140).

En effet, dans la bibliographie initiale - assez impressionnante et inattendue -sont cités les ouvrages de phonétique appliquée les plus importants parus en Europe avec d'autres concernant la phonétique de l'italien, l'art de la lecture et de la diction. Les 
plus connus sont dus à des personnalités fortes qui ont joué un rôle essentiel dans le renouvellement de l'enseignement des langues à la fin du XIX ${ }^{e}$ siècle. Nombreux d'entre eux se retrouvaient dans l'Association Phonétique Internationale. Rappelons, entre autres : Les parlers parisiens. Anthologie phonétique d'Edouard Koschwitz ; le Dictionnaire phonétique français de Michaelis-Passy ; le Manuel du français parlé de Kristopher Nyrop ; Les sons du français et Petite phonétique comparée des principales langues européennes de Paul Passy; Les origines de la prononciation moderne et Exercices pratiques d'articulation et de diction de Théodore Rosset ; Les principes de phonétique expérimentale de l'Abbé Rousse-lot ; le Précis de prononciation française de Rousselot-Laclotte ; Primer of Phonetics de Henri Sweet ; De la prononciation française depuis le commencement du XVI" siècle d'après le témoignage des grammairiens de Charles Thurot; Kleine Phonetik des Englischen, Französischen und Deutchen de Viëtor ; la Méthode pratique, physiologique et comparée de prononciation française d'Adolphe Ziind-Burguet ; L'art de lire d'Emile Faguet, L'art de la lecture d'Ernest Legouvé. Sur l'italien, on trouve De la nasalité en italien de Giulio Panconcelli-Calzia ; Etudes expérimentales de phonétique italienne de F. Josselyn à côté du Nuovo dizionario universale della lingua italiana di Policarpo Petrocchi.

A propos de la transcription phonétique, Ferrante déclare avoir adopté le principe « un seul signe pour un seul son ; un seul son pour un signe " qui est le fondement " phonologique " plusieurs fois réitéré par Passy mais, curieusement, après avoir longtemps hésité entre les deux systèmes concurrents, l'A.P.I. (Passy) et celui de la Société des parlers de France (Rousselot), il a opté pour un alphabet de son invention. En effet, considérant que l'A.P.I. était trop difficile pour les élèves du secondaire et que, en tout cas, les deux posaient des obstacles typographiques difficiles à vaincre en Italie, il a adapté les lettres de l'alphabet-majuscules, minuscules ou renversées, en caractères italiques ou gras (p. 18).

La description des organes de la parole (I. pp. 3-9, sans aucune image), est suivie par la classification des voyelles et des consonnes (II. pp. 11-156). L'auteur indique la façon de mettre en place l'articulation correcte des sons français en contrôlant la position des organes à l'aide de la vue (un petit miroir) ou du toucher.

Par ailleurs, s'étant situé dans une optique contrastive, il attire l'attention sur les fautes typiques les plus tenaces, choquantes à l'oreille et souvent source d'ambiguïtés, par ex. la confusion entre « e » muet et [e], la diphtongaison de la voyelle antérieure labialisée [y], les distorsions des voyelles nasales, et il indique pour chacune des exercices pratiques et des astuces pour les corriger.

Une année plus tard, Ferrante récidive avec une plaquette dont le titre Les sons $d u$ Français rappelle le célèbre ouvrage de Paul Passy mais avec une précision qui ne laisse que peu de doutes sur la teneur du contenu : avec toutes leurs graphies. A l'aide de son système de transcription très discutable, l'auteur présente une liste de 23 voyelles où rien ne permet de distinguer entre les phonèmes et leurs variantes. Aucune définition n'est donnée de ce qu'on entend par voyelles « moyennes», « ouvertes » ou " fermées. » D'abord, pour chaque son (représenté de manière souvent ambiguë) sont données toutes les graphies possibles et impossibles (par exemple, pour les trois variétés de $a$ sont indiquées, entre autres, les graphies : «i»; «y», p. 6). On passe ensuite à illustrer pour chaque lettre tous les sons qu'elle peut représenter dans l'écriture usuelle. Le malheureux lecteur se noie dans un luxe de détails graphiques tout à fait décourageants. Ce travail de compilation minutieuse manque totalement d'originalité. 

lentement » (Emile Faguet, L'Art de Lire). civiques.

\section{Des pistes à suivre} dont la plupart avaient suivi des cours de vacances à l'étranger.

Il nous convie ainsi à suivre deux pistes dans nos recherches : pays tout entier. pas. plutôt rares (en 1905 ils étaient cinq, dont quatre provenant de Sicile).

Quant au Manuale di fonetica francese ad uso delle scuole medie [s.d.], Ferrante y trace les règles de la bonne prononciation (Parte I Pronunzia-Ortografia, parte II Regole particolari di Ortoepia) et consacre la troisième partie à la Diction. Il y propose un ensemble de textes célèbres (entre autres Le maître de philosophie d'après Molière) en orthographe usuelle et en A.RI. : il faut croire que la critique de Paul Passy avait enfin provoqué l'effet voulu. La consigne est toute dans cette citation « Pour apprendre à lire, il faut d'abord lire très lentement et ensuite il faut lire très lentement et, toujours, très

En Appendice on peut lire les Statuts de l'Association Phonétique Internationale (p. 192).

Dans les traités de prononciation que nous avons analysés, l'optique moralisante (dans le choix des lectures, des proverbes, des exemples) tend à s'estomper. Parmi les morceaux proposés par Vincent et Ferrante figurent des auteurs consacrés : Hugo, Nodier, Beaumarchais, Molière, Cyrano de Bergerac, mais aussi des linguistes : Dauzat, Rousselot/Laclotte. Comme l'a bien écrit Nadia Minerva, pendant les XVII ${ }^{\mathrm{e}}$ et XVIII siècles les manuels proposaient un modèle de prononciation... et de morale.

Après l'unification de l'Italie, on croise moins de nobles dans les manuels, l'enseignement se démocratise et l'on voit apparaître les valeurs patriotiques et

Malgré la modestie du gain cognitif et didactique, les ouvrages analysés ont le mérite d'avoir ouvert le débat et peut-être la voie à d'autres applications de la phonétique dans un pays qui avait accumulé beaucoup de retard dans ce domaine.

Tout au long de sa carrière, Lovera a mis l'accent sur l'état de criante nécessité pédagogique qui marquait l'enseignement des langues en Italie : lacunes au niveau de la formation, manques dans la dotation d'appareillages dans les écoles et les universités, absence totale de chaires de philologie moderne et de phonétique. En 1916, face à l'inertie des institutions, il soulignait la contribution des jeunes professeurs de langues,

1. d'une part, creuser du côté des enseignants dont l'histoire reste à écrire. Élaborer une typologie des maîtres de langues (autochtones et/ou francophones), définir leur origine, leur formation (quelle autodidaxie ?), leurs conditions socio-économiques, leurs motivations, leurs publics, les objectifs de leur enseignement. Ce travail d'archéologie n'est qu'amorcé et demande à être étendu à l'ensemble de la Sicile et au

9 Comme c'est souvent le cas aujourd'hui encore, les renouvellements méthodologiques sont dus à l'initiative personnelle des collègues. Les informa-lions dont nous disposons sur les enseignants de l'époque prouvent que certains n'en manquaient certainement

Les quelques inscriptions à l'Association Phonétique Internationale provenant de Sicile autour de 1905 sont-elles le fait du hasard ? Et pourtant les membres italiens étaient 
81 Jacqueline Lillo a montré que pendant le XIX ${ }^{e}$ siècle il faisait bon vivre à Palerme pour les réfugiés étrangers de toutes provenances qui s'étaient recyclés dans renseignement des langues : peu importe les titres académiques pourvu qu'ils soient catholiques et de bonne moralité. La douceur du climat, le caractère accueillant des gens, l'engouement des Palermitains pour le français rendaient Palerme particulièrement attrayante. Nombreux furent donc les Français qui s'y établirent au fil du siècle et jusqu'en 1860 la majorité des enseignants était francophone (Lillo 1992). En allait-il de même pour les autres villes de Sicile?

$82 \mathrm{Si}$ au XVIII ${ }^{\mathrm{e}}$ siècle n'importe qui pouvait s'improviser enseignant par le seul fait d'être Français (Minerva 1992: 8) pour atteindre des objectifs essentiellement mondains (l'art de converser), un siècle plus tard les finalités ont nécessairement changé autant que le monde et la société se sont modifiés : qu'en est-il alors du recrutement des maîtres et des compétences requises? Enfin, il faudrait étudier le rôle joué par les associations professionnelles telle que la Libera Società degl'Insegnanti di Palermo (1866).

2. La deuxième piste consiste à analyser l'impact des cours de vacances sur le public des enseignants de langues en Sicile et en Italie. Quels étaient parmi les plus célèbres cours de l'époque (Marbourg, Paris, Genève, Edimbourg, Grenoble etc.) les pôles d'attraction et pour quelles raisons (voisinage géographique, innovation méthodologique, renommée internationale des enseignants, qualité de l'accueil)?

Les cours d'été de Grenoble ont bénéficié, pendant longtemps, d'une très bonne réputation en Italie. Les Italiens y accouraient nombreux dès le début, le Ministère de l'Instruction Publique s'y était intéressé et la presse s'était fait l'écho de ce succès.

Les villes allemandes n'étaient pas moins actives (n'oublions pas les initiatives de Marbourg en faveur de l'italien).

3. J'ajouterai une troisième voie qui me paraît prometteuse : l'analyse détaillée des petites revues éphémères, des bulletins des catégories professionnelles et même de la presse de l'époque qui fournit souvent des informations et des témoignages précieux. Les revues constituent un canal privilégié dans la diffusion des idées et une tribune de débat souvent très animée par des confrontations à distance. Elles mériteraient d'être analysées de plus près.

Des documents " pauvres » tels que les petites annonces ont déjà retenu l'attention de certains d'entre nous (Londei 1991).

89 A maints égards, comme je me suis efforcée de le montrer tout au long de mon exposé, le cas de la Sicile me parait particulièrement intéressant pour le chercheur; il fourmille de pistes à explorer et nous invite à approfondir les causes de l'attachement passionné des Siciliens à la langue et à la culture françaises.

90 Les quelques éléments que j'ai livrés dans ma communication sont des propositions d'interprétation ouvertes à la discussion qui permettent de baliser de nouvelles recherches. 


\section{Matériaux (par ordre chronologique)}

BONGIOANNI, Luigi (1881) : Regole di retta pronuncia francese dettate ai suoi alunni dal Professore L. B. E seguite da una raccolta di frasi italiane e francesi.Matera, Tipografia Conti.

SIMONETTI, Pio (1899) : Lezioni di pronunzia ed ortografia francese secondo il metodo fonetico naturale. Naples, Circolo Poliglottico Napoletano (3e éd.).

DEODATO, Jean (1897) : Textes français pour tous les genres de composition [...] précédé d'un traité complet de prononciation. Messine, Toscano.

FIORETTO, Giuseppe (1903) : La pronunzia francese secondo il metodo naturale. Vicence, G. Galla.

CONTE, Giuseppe (1906) : Trattato di pronuncia francese a base fonetica (préf. de Romeo Lovera). Molfetta, M. Conte.

FERRANTE, Alberto (1912) : Petite phonétique de la langue française à l'usage des Italiens. Caltanissetta, Panfilo Castaldi - S. Petrantoni.

FERRANTE, Alberto (1913) : Les sons du français avec toutes leurs graphies. Caltanissetta, Stab. Tip. S. Petrantoni.

FERRANTE, Alberto (s.d.) : Manuale di fonetica francese ad uso delle scuole medie. Palerme, G. Priulla Salemi, Coli. « Testi scolastici Priulla. »

DI GREGORIO, G. (1915) : La riforma ortografica dell'inglese, del francese e dell'italiano. Palerme.

FIORETTO, Giuseppe (1922) : Extrait de La phonétique par le gramophone. Les sons du français. Méthode naturelle. A l'usage des écoles et des familles italiennes el étrangères. Vérone, Remigio Cabianca. Nouvelle édition.

FOULCHE-DELBOSC, R. (s.d.) : Echo du français parlé. Con vocabolario speciale di Romeo Lovera. LeipzigTurin-Palerme, C.Clausen.

VINCENT, Carlo (1906) : Manuale di fonetica francese. Catania coi tipi dell'autore.

ZANARDELLI, Tito (s.d.) : II francese scritto e parlato nei suoi primi elementi. Trattato di pronunzia e compendio di grammatica esposti con nuovi criteri e metodo moderno. Milan-Palerme-Naples, Sandron.

\section{Sources secondaires}

AA.VV. (2000) : Soliloquio d'un fauno. Mallarmé tradotto da Vincenzo Palumbo, Prefazione di Mario Luzi, Testo a cura di Maria Gabriella Adamo. Con un saggio di Sergio e Massimo Palumbo. Nota di Maria Teresa Giaveri. Pise, Éditions ETS

ADAMO, Maria Gabriella (2001) : «Su alcune traduzioni del Monologue d'un faune : Mallarmé et « le vent de Sicile ", in AA.VV.,Mallarmé: un secolo di poesia. Actes du Congrès de la SUSLLF. Naples 11-12 novembre 1999, La Città del Sole.

AUROUX,Sylvain éd. (2000) : Histoire des idées linguistiques, Vol. III, L'Hégémonie du Comparatisme. Liège, Mardaga. 
AUROUX, Sylvain, KOERNER, E. F.K. et alii éds (2001) :Histoire des sciences du langage, Tome 2. XXVIII, L'étude de la parole et des langues non écrites pendant le XIX ${ }^{e}$ siècle et sa continuation au XX siècle. Berlin - New York, W. de Gruyter.

BATTAGLIA, Rosario, FEDELESanti, LO CURZIO M. (éds) (1997) : Messina negli anni Venti e Trenta. Una città meridionale tra stagnazione e fermenti culturali. Istituto di Studi Storici Gaetano Salvemini. Messine, Sicania.

BOCHICCHIO, Franco (1993) : «L'enseignement de la langue française et le système scolaire italien de 1860 à 1913 ", Documents SIHFLES 12, 16-24.

BRETHOME, Jacques (1996) : Les professeurs d'allemand des lycées en France, 1850-1880. Thèse de Doctorat, Univ. de Nantes. Directeur J.-P. Barbe.

CAPPELLO, Maria Luisa (1994) : « L'enseignement de la prononciation du français aux XVIII ${ }^{\mathrm{e}}$ et XIX ${ }^{\mathrm{e}}$ siècles en Italie. » Documents pour l'histoire du français langue étrangère ou seconde 14, 144-150. Paris, SIHFLES.

CAPPELLO, Maria Luisa (1995) : « Le rôle de l'enseignement des langues vivantes depuis la « Scuola Superiore di Commercio con Banco-modello » (1875)jusqu'à la Facoltà di Lingue Straniere (1969) à Bari (Italie) ». Documents pour l'histoire dit français langue étrangère ou seconde 15, 67-77. Paris, SIHFLES.

CAPPELLO, Maria Luisa (1997) : « Les professeurs italiens face aux innovations de l'A.P.I. ». Documents pour l'histoire du français langue étrangère ou seconde 19, 198-205. Paris, SIHFLES.

CIGADA, Sergio (1985) : «Italia e Francia nella letteratura di fine secolo. » Il Novecento letterario in Italia, vol. I. Milan, Vita e Pensiero, 9-40.

DARCHINI, Gaetano (1908) : « I corsi estivi di Grenoble. » La Cultura, Rivista critica fondata da Ruggero Borghi. Rome, 15 février 1908, 130-132.

D’ELIA, Raffaele (1908) « I corsi estivi di Grenoble. » Bollettino di Filologia Moderna, mars 1908, 60-62.

GALAZZI, Enrica (1987) : « Paul Passy : la fonetica al servizio dell'insegnamento delle lingue», in Tre secoli di glottodidattica (a cura di B.Cambiaghi). Scuola e lingue moderne, 1987 1/2, 15-18.

GALAZZI, Enrica (1991) : « La méthode phonétique dans l'enseignement du FLE en Italie à travers la lecture du 'Maître phonétique' et du 'Bollettino di filologia moderna' », in MANDICH PELLANDRA éds, (1991), 277-300.

GALAZZI,Enrica (1992) : « 1880-1914. Le combat des jeunes phonéticiens : Paul Passy. » Cahiers Ferdinand de Saussure 46, 115-129.

GALAZZI,Enrica (1995) : « Phonétique / Université / Enseignement des langues à la fin du XIX siècle. » Histoire Epistemologie Langage, XV1I/1, 95-114.

GALAZZI, Enrica (1997) : «Physiologie de la parole et enseignement de la prononciation fin XIX ${ }^{\mathrm{e}} /$ début $\mathrm{XX}^{\mathrm{e}}$ siècles. Documents pour l'histoire du français langue étrangère ou seconde 19, 166-183. Paris, SIHFLES.

GALAZZI, Enrica (1997) : « Théodore Rosset : une méthode originale pour l'enseignement de la prononciation française à l'Université de Grenoble dès 1904 ", Documents pour l'histoire du français langue étrangère ou seconde 20, 37-53. Paris, SIHFLES.

GALAZZI, Enrica (2000) : «L'Association Phonétique Internationale », in AUROUX éd. (2000), 499-516. 
GALAZZI, Enrica (2001) : «Physiologie de la parole et phonétique appliquée au XIX et au début du XX ${ }^{\mathrm{e}}$ siècle », in AUROUX-KOERNER et alii éds (2001), 1485-1498.

HAMMAR, Elisabet (1991) : «La Française. » Mille et une façons d'apprendre le français en Suède avant 1807. Upsala, Acta Universitatis Upsaliensis.

KOK ESCALLE, Marie Christine, MELKA, Francine éds. (2001) : Changements politiques et statut des langues. Histoire et epistemologie 1780-1945. Amsterdam, Rodopi.

LILLO, Jacqueline (1992) : « Les professeurs de français à Palerme de 1800 à 1860. » Documents pour l'histoire du français langue étrangère ou seconde 9, 10-17. Paris, SIHFLES.

LILLO, Jacqueline (1993) : « Un avis de concours à un poste d'enseignement de français et d'allemand à l'Institut technique de Palerme en 1865. » Documents pour l'histoire du français langue étrangère ou seconde 12, 25-31. Paris, SIHFLES.

LILLO, Jacqueline (1995) : « Recrutement des professeurs de langue à Palerme tout de suite après l'unification de l'Italie (1860-1878) », Documents pour l'histoire du français langue étrangère ou seconde 15, 3-159. Paris, SIHFLES.

LILLO, Jacqueline (1999) : « Les professeurs de français à Palerme de 1860 à 1900 », Documents pour l'histoire du français langue étrangère ou seconde 23, 453-480. Paris, SIHFLES.

LONDEI, Danielle (1991) : «'Offresi / Cercasi professore di francese'. Les petites annonces à Bologne au XIX siècle », in MANDICH - PELLANDRAéds. (1991), 211-220.

LOVERA, Romeo (1896) : «L'insegnamento del francese in Italia », in Fra lingue e letterature straniere. Turin, Clausen, 97-1 10.

LOVERA, Romeo (1907) : Corso di lingua francese a base intuitiva. Anno I. Palerme, Libreria editrice del Bollettino di Filologia Moderna.

LOVERA, Romeo (1916) : L'enseignement du français à l'Institut des hautes études commerciales de Turin, Turin, Imp. Degli Artigianelli.

MANDICH, Anna Maria, PELLANDRA, Carla éds. (1991) : Pour une histoire de l'enseignement du français en Italie. Actes du Colloque de Parme, 14-16 juin 1990. Documents pour l'histoire du français langue étrangère ou seconde 8. Paris, SIHFLES.

MESCHONNIC, Henri (2000) : «L'avenir est au sens du langage », in Français de l'avenir et avenir du français. Les Cahiers du CIEP. Paris, Didier, 115-121.

MINERVA, Nadia (1996) : Manuels, Maîtres, Méthodes. Repères pour l'enseignement du français en Italie. Bologne, CLUEB.

MINERVA, Nadia, PELLANDRA, Carla (1991) : Insegnare afrancese in Italia. Repertorio dì manuali pubblicati dal 1625 al I860. Bologne, Patron editore.

MOLONIA, Giovanni (1997): « Editorìa, riviste, giornali », in BATTAGLIA - FEDELE -

LOCURZIO(1997), 507-525.

PAGLIERINI(1915) : Catalogo generale della libreria italiana, dall'anno 1847 al 1899. Milan, Assoc. Tipogr. Libr. Ital.

PELLANDRA, Carla (1992) : «Les livres d'un professeur de français d'autrefois : le Fonds Ferretti du Dipartimento di Lingue e Letterature Straniere Moderne de Bologne. » Documents pour l'histoire du français langue étrangère ou seconde 9, 24-29. Paris, SIHFLES. 
PELLANDRA, Carla (1995) : «Pietro Toldo et l'institution de la première chaire de langue et de littérature françaises dans l'université italienne ». Documents pour l'histoire du français langue étrangère ou seconde 15, 116-128. Paris, SIHFLES.

PUREN, Christian (1988) : Histoire des méthodologies de l'enseignement des langues. Paris, Nathan-Clé International.

RESTORI, Antonio (1907) : « Relazione letta nell'aula magna addi' 5 novembre 1906. » Per l'inaugurazione dell'anno accademico 1906-1907, in Annuario della R. Università di Messina, 1906-1907. Messine, Tipografia D’Angelo, 5-10.

ROMANO, Andrea (1997) : «L'Università degli studi di Messina negli anni Venti e Trenta del Novecento », in BATTAGLIA - FEDELE - LO CURZIO, 405-420.

ROSSIGNOTTI, Giovanna (1991) : « La didattica della lingua francese attraverso l'analisi del 'Bollettino di Filologia Moderna' (1894-95; 1901-1909) ». Documents pour l'histoire du français langue étrangère ou seconde 8, 265-276. Paris, SIHFLES.

SANTANGELO, Giovanni (1975) : «Studiosi di letteratura francese in Sicilia tra Ottocento e Novecento. » Archivio storico siciliano, s. IV, vol. I, 1-79.

VACCA, Salvatore (2001) : Angelico Lipani e la tradizione cappuccina in Sicilia. Ed. Salvatore Sciascia.

VITALE, Filomena (2001) : « Le rayonnement du français dans le Royaume de Naples de 1799 à $1860 »$, in KOK ESCALLE - MELKA(2001), 75-86.

\section{RÉSUMÉS}

Il est tout à fait instructif de porter un regard diachronique sur les liens qui existent entre l'étude de la phonétique et l'enseignement de la langue française. Le cas de la Sicile entre la fin du XIXe siècle et le début du XXe est sur ce point particulièrement fécond puisqu'on y publie différents traités consacrés à la prononciation du français. Quelles que soient les réserves méthodologiques et scientifiques que l'on peut faire sur ces ouvrages, ceux-ci ont eu, en Italie, le mérite décisif d'avoir ouvert la réflexion sur l'importance de la phonétique dans la linguistique et la didactique modernes.

There is certainly a lot to be learned from a diachronic overview of the links between the study of phonetics and the teaching of French. The case of Sicily between the end of the 19th century and the beginning of the 20th is especially fruitful because several treatises on French pronunciation were published there. Whatever methodological and scientific reservations may be held about these works, they were decisive in opening a debate new to Italy, with reflections on the importance of phonetics in linguistics and language teaching.

\section{INDEX}

Mots-clés : Linguistique, didactique, enseignement du français, phonétique, Sicile, XIXe siècle, XXe siècle

Keywords : Linguistics, language teaching, phonetics, Sicily, 19th century, 20th century 
AUTEUR

ENRICA GALAZZI

Université catholique, Milan 